\title{
Spatial Pattern Formation During Aggregation of the Slime Mould Dictyostelium discoideum
}

\author{
Catelijne van Oss $\dagger$, Alexandre V. Panfilov, Pauline Hogeweg, \\ Florian Siegert $\$$ AND CORnelis J. WeIJER $\$ \S$ \\ Department of Theoretical Biology, Utrecht University, Padualaan 8, 3584 CH Utrecht, \\ The Netherlands and $\ddagger$ the Zoologisches Institut, Universität München, Luisenstr. 14, \\ 80333 München 2, Germany
}

(Received on 5 December 1995, Accepted in revised form on 1 April 1996)

\begin{abstract}
Stream formation and spiral wave behaviour during the aggregation of Dictyostelium discoideum $(D d)$ are studied in a model based on the Martiel-Goldbeter equations for cAMP relay, combined with chemotactic motion of $D d$ cells. The results show that stream formation occurs if the turnover rate of intracellular cAMP is increased. This increase in the turnover rate of $\mathrm{cAMP}_{[\text {[in] }}$ leads to a dependence of the speed of the cAMP wave on the cell density. We propose that this dependence of wave speed on cell density is the underlying mechanism for stream formation. Besides stream formation, increasing the turnover rate of $\mathrm{cAMP}_{[i n]}$ also results in a spiral wave period that decreases during aggregation, a phenomenon that is commonly observed in situ.

Furthermore, the dependence of wave speed on cell density is measured empirically. The speed of the cAMP wave is found to decrease as the wave travels from high to low cell density. This indicates that in situ, wave speed does depend on cell density.
\end{abstract}

(C) 1996 Academic Press Limited

\section{Introduction}

Dictyostelium discoideum $(D d)$ is widely used to study multicellular morphogenesis. In normal conditions, Dd consists of unicellular amoebae that feed on bacteria in the soil. When food becomes scarce, the amoebae enter an aggregation phase, leading to the formation of a multicellular organism (slug) and finally to a fruiting body. The spores can germinate and give rise to amoebae, which completes the life cycle (Loomis, 1982). During aggregation, amoebae communicate by periodically producing and relaying cAMP signals. The resulting cAMP waves, initiated by amoebae in the aggregation centre, propagate as concentric waves or rotating spirals. The amoebae

\footnotetext{
$\uparrow$ Author to whom correspondence should be addressed. Temporary address until November 1996: Theoretische Biologie, Universität Bonn, Kirschallee 1-3, D-53115 Bonn, Germany.

E-mail: cvo@binf.biol.ruu.nl

§Present address: Dept. of Anatomy and Physiology, Old Medical School, University of Dundee, Dundee DD1 4HN, Scotland, U.K.
}

react chemotactically to cAMP and move to the aggregation centre. During this process, the cells form radial branching structures (streams). The streams gradually disappear, and the cells collect in the aggregation centre (Devreotes, 1982; Tomchik \& Devreotes, 1981).

Various aspects of $D d$ aggregation have been studied mathematically. Keller \& Segel (1970) proposed that the onset of aggregation might be viewed as a breakdown of stability. Chemotaxis in one dimension was studied by Parnas \& Steel (1977, 1978). Aggregation and stream formation were studied in the models of Mackay (1978) and Vasieva et al. (1995). Both models include discrete cells that can be in one of three states: rest, excited or refractory, every excited cell moving to the nearest excited cell. Kessler \& Levine (1993) combined a comparable phenomenological-rule model with diffusion of CAMP and proposed that the stream formation was due to instability of the combined signalling-chemotaxis system (also see Levine \& 
Reynolds, 1991). Recently, Höfer et al. (1995a, b) have reported that in their model, stream formation is also due to a chemotaxis-driven wavefront instability. Vasiev et al. (1994) proposed a reactiondiffusion model consisting of the Fitzhugh-Nagumo equations for the process of cAMP production and a continuous density variable. In this model, stream formation is due to the density-dependent wave speed, cAMP waves travelling faster in regions with higher cell density.

The process of cAMP signalling without chemotaxis has been studied by Tang \& Othmer (1994), Monk \& Othmer (1990) and by Martiel \& Goldbeter (1987) in models based on experimental data. The latter model was used by Tyson et al. (1989) to study spiral waves on a plane, assuming a homogeneous amoebae distribution. In the present paper, we extend the Martiel-Goldbeter model to include chemotaxis and study the process of aggregation induced by a point source and by a spiral wave. We show that in this model, stream formation is dependent on the dynamics of intracellular cAMP. We also investigate the consequences of two different chemotaxis equations for the streaming pattern.

The model results indicate that the relation of wave speed to cell density is important in stream formation. We measured this relation in an experiment, and compare the findings with the model results.

\section{The Model}

\subsection{METHODS}

The model describes discrete $D d$ cells which can produce cAMP and move towards a cAMP gradient. We used the Martiel-Goldbeter equations, which specify the following mechanism for signal relaying: $D d$ cells have a transmembrane cAMP receptor which can be in either of two states: active or inactive. In the active state, the receptor can bind $\mathrm{cAMP}_{[\mathrm{ex}]}$, thereby stimulating the synthesis of cAMP inside the cell. $\mathrm{cAMP}_{[\text {in] }}$ is transported to the outside of the cell, where it stimulates the cAMP receptor and thereby closes the positive feedback loop. The cAMP receptor changes to the inactive state as a result of prolonged exposure to high $\mathrm{cAMP}_{[\mathrm{ex}]}$ concentrations. This limits the cAMP production and makes the cell refractory. Meanwhile, $\mathrm{cAMP}_{[\mathrm{ex}]}$ decays.

To these equations [including the diffusion operator on $\mathrm{cAMP}_{[\mathrm{ex}]}$, as added by Tyson et al. (1989)] we add discrete cells in the following way. The position of amoeba $i$ in space is given by $\mathbf{R}_{\mathbf{i}}(t)=$ $\left(x_{i}(t), y_{i}(t)\right)$. We use the delta function $\delta\left(\mathbf{r}-\mathbf{R}_{\mathbf{i}}(t)\right)$, where $\mathbf{r}=(x, y) . \delta\left(\mathbf{r}-\mathbf{R}_{\mathbf{i}}(t)\right)$ equals " 1 " if $\mathbf{r}=\mathbf{R}_{\mathbf{i}}(t)$ and equals " 0 " otherwise. Every term in the equations is multiplied by this delta function, except the diffusion term. In this way the Martiel-Goldbeter equations apply at the location of an amoeba, whereas in empty space only the diffusion term applies. Thus, we assume that decay of cAMP [ex] occurs only at the location of a cell, although the model results are scarcely affected even if there is a little extracellular cAMP decay. The equations (written in dimensionless form) become:

$$
\begin{aligned}
& \varepsilon_{r} \frac{\partial \rho(\mathbf{r}, t)}{\partial \mathrm{t}}=[-f_{1}(\gamma) \rho(\mathbf{r}, t) \\
&\left.+f_{2}(\gamma)(1-\rho(\mathbf{r}, t))\right] \sum_{i=0}^{N} \delta\left(\mathbf{r}-\mathbf{R}_{\mathbf{i}}(t)\right) \\
& \varepsilon_{i} \frac{\partial \beta(\mathbf{r}, t)}{\partial t}=\left[s_{1} \phi(\rho, \gamma)-\beta(\mathbf{r}, t)\right] \sum_{i=0}^{N} \delta\left(\mathbf{r}-\mathbf{R}_{\mathbf{i}}(t)\right) \\
& \frac{\partial \gamma(\mathbf{r}, t)}{\partial t}=D \nabla^{2} \gamma(\mathbf{r}, t)+\frac{1}{\varepsilon_{e}}\left[s_{2} \beta(\mathbf{r}, t)\right. \\
&\quad-\gamma(\mathbf{r}, t)] \sum_{i=0}^{N} \delta\left(\mathbf{r}-\mathbf{R}_{\mathbf{i}}(t)\right)
\end{aligned}
$$

where

$$
\begin{aligned}
f_{1}(\gamma)=\frac{1+\kappa \gamma}{1+\gamma} ; & f_{2}(\gamma)=\frac{L_{1}+\kappa L_{2} c \gamma}{1+c \gamma} ; \\
& \Phi(\rho, \gamma)=\frac{\lambda_{1}+Y^{2}}{\lambda_{2}+Y^{2}} ; \quad Y=\frac{\rho \gamma}{1+\gamma} .
\end{aligned}
$$

$\rho(\mathbf{r}, t)$ is the fraction of cAMP receptor in active state, $\beta(\mathbf{r}, t)$ is intracellular cAMP and $\gamma(\mathbf{r}, t)$ is extracellular cAMP, $N$ is the number of $D d$ cells.

To integrate this set of partial differential equations we used the explicit Euler method with space step $=0.01$ and time step $=0.001$. The timescale is $8.3 \mathrm{~min}$ and the space scale is $4.5 \mathrm{~mm}$, which is the same as in Tyson et al. (1989). Typically, the size of one cell is the size of the space step, and to do our simulations within reasonable computer time, the size of our cells is $45 \mu \mathrm{m}$. This is several times larger than the size of real amoebae. However, we checked the main results of our paper [Fig. 1(a and b)] for space step $=2 \cdot 10^{-4}$, which corresponds to $10 \mu \mathrm{m}$, and found that the results are similar. The typical grid size is $200 \times 200$ mesh points (unless mentioned otherwise in the figure captions), which corresponds to a field of $0.9 \times 0.9 \mathrm{~cm}$. To mimic an isolated aggregation field, Dirichlet boundary conditions (variables $=0$ at the boundary) are employed throughout. The results are 
not affected by the use of "no flux" boundary conditions. In this paper, cell density is given in (surface covered by amoebae)/(total surface). The typical cell density used is $45 \%$. If the cell size is $45 \mu \mathrm{m}$, this means the density is $2.2 \times 10^{4} \mathrm{cells} / \mathrm{cm}^{2}$, whereas in the case of cell size $=10 \mu \mathrm{m}$, the density is $4.5 \times 10^{5} \mathrm{cells} / \mathrm{cm}^{2}$. The parameter values are the same as in Martiel \& Goldbeter (1987) and Tyson et al. (1989) (set c), namely: $c=10 ; s_{1}=950 ; s_{2}=$ $0.05 ; \lambda_{1}=10^{-3} ; \lambda_{2}=2.4 ; \kappa=18.5 ; \quad \varepsilon_{e}=0.01 ; \varepsilon_{i}=$ $0.019 ; \quad \varepsilon_{r}=1 ; \quad L_{1}=10 ; \quad L_{2}=5 \times 10^{-3} ; \quad \mathrm{D}=0.01$, unless mentioned otherwise.

If internal cAMP dynamics is very fast, $\beta$ can be assumed to be at quasi-steady-state and eqn (1) reduces to:

$$
\begin{aligned}
& \varepsilon_{r} \frac{\partial \rho(\mathbf{r}, t)}{\partial t}=\left[-f_{1}(\gamma) \rho(\mathbf{r}, t)+\right. \\
& \left.f_{2}(\gamma)(1-\rho(\mathbf{r}, t))\right] \sum_{i=0}^{N} \delta\left(\mathbf{r}-\mathbf{R}_{\mathbf{i}}(t)\right) \\
& \frac{\partial \gamma(\mathbf{r}, t)}{\partial t}=D \nabla^{2} \gamma(\mathbf{r}, t)+\frac{1}{\varepsilon_{e}}[s \Phi(\rho, \gamma) \\
& -\gamma(\mathbf{r}, t)] \sum_{i=0}^{N} \delta\left(\mathbf{r}-\mathbf{R}_{\mathbf{i}}(t)\right) .
\end{aligned}
$$

We will use both eqns (1) and (2) in our simulations.

Dictyostelium cells orientate to the cAMP gradient, moving 10-20 $\mu \mathrm{m}$ (approximately one cell length) per wave (Devreotes, 1982). We can describe chemotaxis by the following equation:

$$
\frac{\mathrm{d} \mathbf{R}_{\mathbf{i}}(t)}{\mathrm{d} t}=\left.\mu \nabla \gamma(\mathbf{r}, t)\right|_{\mathbf{R}_{\mathbf{i}}(t)}
$$

where

$$
\nabla \gamma(\mathbf{r}, t)=\left(\frac{\partial \gamma(\mathbf{r}, t)}{\partial x}, \frac{\partial \gamma(\mathbf{r}, t)}{\partial y}\right)
$$

In this case, cells adjust both their direction and their speed to the cAMP gradient. However, it might be that $D d$ cells use only the directional information of the gradient, and move with their own intrinsic speed $\mu$ :

$$
\frac{\mathrm{d} \mathbf{R}_{\mathbf{i}}(t)}{\mathrm{d} t}=\left.\mu \frac{\nabla \gamma(\mathbf{r}, t)}{\|\nabla \gamma(\mathbf{r}, t)\|}\right|_{\mathbf{R}_{\mathrm{i}}(t)}
$$

Although eqn (3) is simpler in its mathematical description, it is possible that for a biological cell, it is simpler to move according to eqn (4). In the latter case, the cell only adapts its direction to external influences and not its speed. Much work has been done on chemotaxis (see for instance Wessels et al. 1992; Steinbock et al. 1991; Varnum-Finney et al. 1988; for reviews on $D d$, see Devreotes, 1989; Devreotes \& Zigmond, 1988; Newell \& Liu, 1992; and on leukocytes, see Wilkinson \& Haston, 1988). However, it is not clear whether $D d$ amoebae use both the direction and the steepness of the cAMP gradient for chemotaxis [as in eqn (3)], or use only the directional information [as in eqn (4)]. We will use first eqn (4) and then eqn. (3) and discuss their impact on the model results.

The parameter $\mu$ is a stepwise function which equals 0 if one of the following conditions is satisfied:

(1) the amoebae is refractory, i.e. $\rho(\mathbf{r}, t)<0.7$. In other words, to explain the fact that amoebae do not respond to cAMP gradients in the back of the cAMP wave, we assume that the chemotactic apparatus becomes desensitized as a result of prolonged $\mathrm{cAMP}_{[\mathrm{ex}]}$ stimulation [a more elaborate discussion on this is given in Höfer et al. (1994)].

(2) $\nabla \gamma(\mathbf{r}, t)<\theta$, where $\theta$ is a threshold value preventing motion towards very small gradients. Such a threshold is observed empirically in for instance Fisher et al. (1989).

(3) $\mathbf{R}_{\mathbf{i}}(t+\Delta t)=\mathbf{R}_{\mathrm{j}}(t+\Delta t), i=1, \ldots, N_{i} \neq j$; and $\Delta t$ is the time step in the simulations. Two amoebae cannot be at the same position at the same time.

Otherwise, $\mu=20$ unless eqn (3) is used, in those cases $\mu=1$. The parameter $\theta=1$ in any case.

\subsection{RESULTS}

\section{Stream formation}

Using the model defined by eqns (1) and (4) and the parameter values stated above, we find that the cAMP wave disappears if the cell density drops below $60 \%$. To increase the wave amplitude and thus improve wave propagation, we have to increase the excitability of the medium. This can be done by either decreasing $\varepsilon_{e}$ or $\varepsilon_{i}$ (by so doing we do not change any other properties of the Martiel-Goldbeter equations, such as number, location and stability of equilibrium points). Decreasing $\varepsilon_{e}$ results in a faster turnover rate of $\mathrm{cAMP}_{[\mathrm{ex}]}$, whereas decreasing $\varepsilon_{i}$ speeds up the dynamics of $\mathrm{CAMP}_{[\mathrm{in}]}$. Figure 1(a) shows a representative simulation with $\varepsilon_{e}$ decreased five times $\left(\varepsilon_{e}=0.002\right.$, $\left.\varepsilon_{i}=0.019\right)$. The amoebae move towards the centre and form a dense spot. In contrast to experimental observations, streams do not form. Neither a smaller decrease, nor a further decrease in $\varepsilon_{e}$ results in stream 
formation. Wave speed $(v)$ as a function of cell density $(d)$ is plotted in Fig. 1(c) (solid line); wave speed is nearly constant when cell density ranges from $100 \%$ to $15 \%$.

If instead $\varepsilon_{i}$ is decreased five times $\left(\varepsilon_{e}=0.01\right.$, $\left.\varepsilon_{i}=0.0038\right)$, streams do form [Fig. 1(b)]. In contrast to the previous parameter setting [Fig. 1(c), solid line] wave speed depends on cell density [Fig. 1(c), dotted line]. A further decrease in $\varepsilon_{i}$ ultimately reduces the model defined by eqns (1) and (4) to the model defined by eqns (2) and (4). In the latter case, the difference in wave speed at low and at high density is larger, the streaming pattern arises earlier and streams are more pronounced. These observations suggest that the dependence of wave speed on cell density is important in stream formation (see Discussion).

Another factor which could be important in stream formation is the cell speed. We investigated this by varying the cell speed parameter $\mu$ from 1 to 30 . It appears that the occurrence of stream formation is not affected, although aggregation takes longer if $\mu$ is small.

In the previous simulations, we assumed that cells move in the direction of the gradient at their own, constant speed $\mu$ [eqn (4)]. However, it might be that $D d$ cells move faster if the gradient is steeper, which is expressed in eqn (3). When using the model defined by eqns (1) and (3) and $\varepsilon_{e}=0.002, \varepsilon_{i}=0.019$, there is

(a)

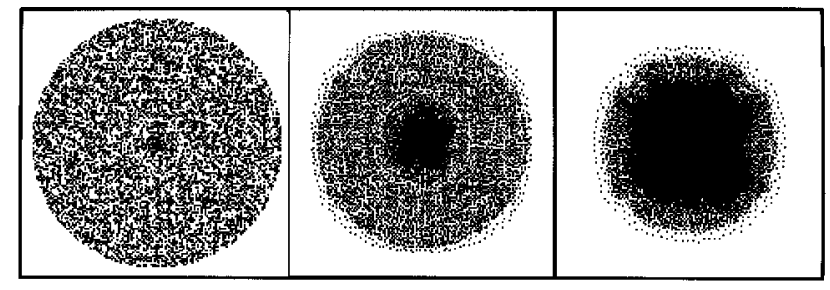

(b)

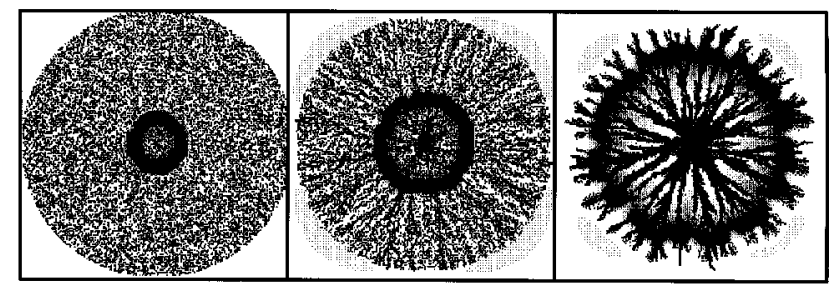

(c)

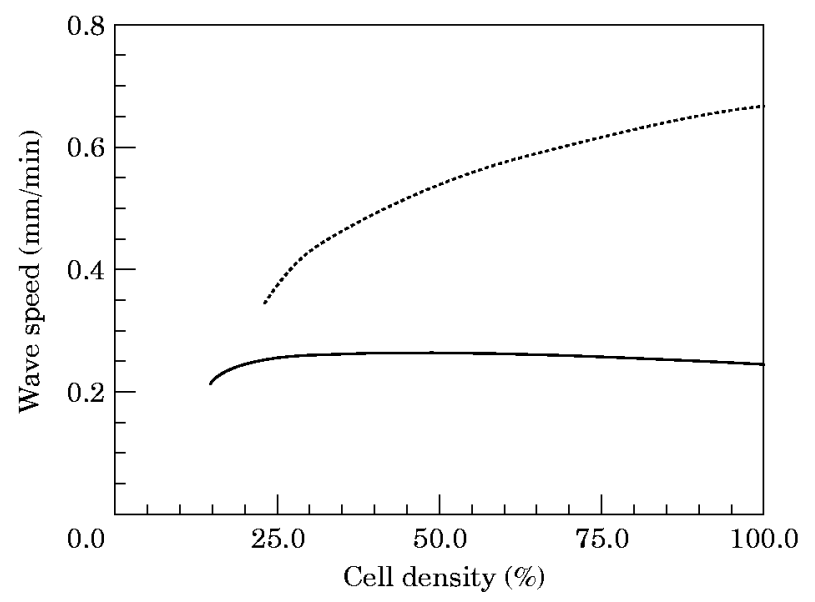

Fig. 1. (a) Aggregation in the model defined by eqns (1) and (4), with $\varepsilon_{e}=0.002 ; \varepsilon_{i}=0.019$ at successive points in time. Time $=5$, 50 and $140 \mathrm{~min}$, respectively. Dictyostelium cells are shown in black, high $\gamma$ values are superposed in grey. White is empty space. Initially, the cells are randomly distributed. The field is artificially stimulated by periodically raising $\gamma$ in the centre of the field, period $=8.3 \mathrm{~min}$; $\mu=20$; field size is $150 \times 150$ mesh points, all other parameters as in Section 2.1. (b) Aggregation in model (1)(4) with $\varepsilon_{e}=0.01 ; \varepsilon_{i}=0.0038$ Time $=5,45$ and $170 \mathrm{~min}$, respectively. Period of stimulation is $8.3 \mathrm{~min} ; \mu=20$. (c) Wave speed vs. cell density in the parameter setting of Fig. 1(a) (solid line) and that of Fig. 1(b) (dotted line). Cell density is defined as (surface covered by amoebae)/(total surface). For several values of the cell density $(100,90,80,70,60,50,40,30,20,19,18,17,16,15,14,13 \%$ for the solid line, 100,90,80,70, 60, 50, 40, 30, $25,24,23,22 \%$ for the dotted line), the following numerical experiment is done. Cells are randomly distributed with a given density, chemotaxis is blocked $(\mu=0)$ and the speed of a plane wave is determined by measuring the time of arrival of the cAMP wave at two assay points a known distance apart. The curves are not influenced by the use of eqn (3) or (4), since $\mu$ is set to zero anyway. 
(a)

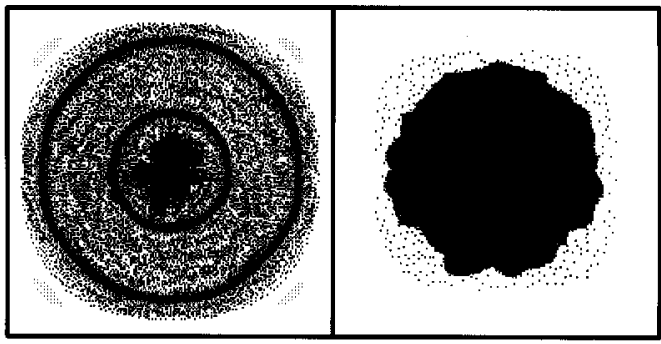

(b)

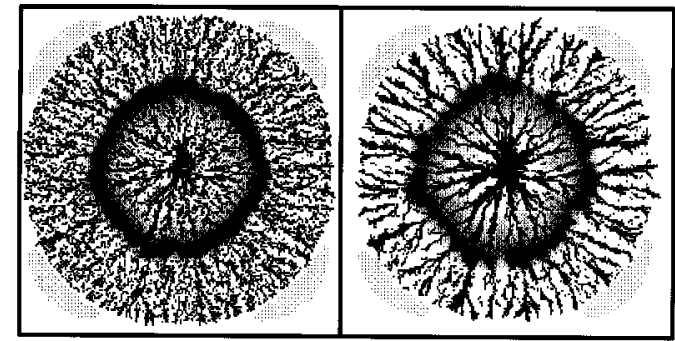

FIG. 2. Aggregation in the model defined by eqns (1) and (3), with $\varepsilon_{e}=0.002 ; \varepsilon_{i}=0.019$. Time $=80$ and 410 min, respectively. Period of stimulation is $8.3 \mathrm{~min} ; \mu=1$, all other parameters as in Section 2.1. (b) Aggregation in model (1)(3), with $\varepsilon_{e}=0.01 ; \varepsilon_{i}=0.0038$. Time $=55$ and $130 \mathrm{~min}$, respectively, the picture at time $=5 \mathrm{~min}$ is similar to that in Fig. 1(b). All other parameters as in Fig. 2(a).

still no stream formation [Fig. 2(a)], although small clusters of high cell density appear. With $\left(\varepsilon_{e}=0.01\right.$, $\left.\varepsilon_{i}=0.0038\right)$, streams arise earlier, are thinner and more distinct [Fig. 2(b)] than in Fig. 1(b). It seems that the use of eqn (3) enhances the process of stream formation. This enhancement of the cell streaming is probably due to cell clustering (see Fig. 2). At a locally higher cell density, more cAMP is produced, resulting in a steeper gradient. When governed by eqn (3), cells move faster in a steeper gradient. They will therefore move faster towards a region of high cell density than towards low density regions and can thus form clusters of high cell density. These clusters enhance the local differences in cell density, which leads to more pronounced streams (see the mechanism to stream formation proposed in the Discussion).

In our model, two cells cannot be at the same place at the same time (see Section 2.1 last paragraph). This is a good approximation of the first stages of aggregation, but when $D d$ cells pile up in streams and in the centre, our model is less realistic. This is particularly visible in the cases where streams are formed: the model cells get stuck in the streams and do not aggregate further. However, simulations show that if this assumption is relaxed and more than one cell can be in one position (Van de Merwe, 1995), this problem is solved. The model cells gather in the streams, the streams become shorter and shorter, and finally all cells are collected in the centre.

\section{Spiral wave behaviour}

Aggregation in $D d$ occurs either as a result of concentric waves or due to a spiral wave. To study aggregation due to a spiral wave, we used the model defined by eqns (2) and (4). In the parameter setting as in Section 2.1, the tip of the spiral wave shows random drift and finally disappears. The drift is most probably due to the increasing heterogeneity of the medium caused by stream formation, since in the model defined by eqns (1) and (4) with $\varepsilon_{e}=0.002$, $\varepsilon_{i}=0.019$, where no streams are formed, spiral waves are stable. This view is supported by the fact that Tyson et al. (1989) and Monk \& Othmer (1990), who used a homogeneous cell distribution in their model, did not observe this random drift of the spiral tip. To increase the stability of a spiral wave, we can use two methods: either decrease the refractory period of the cells by decreasing the parameter $\varepsilon_{r}$, or introduce random motion of the cells (see below). Figure 3(a) shows a typical simulation with $\varepsilon_{r}=0.71$ without random motion. Initially, the tip of the spiral wave wanders, but only over a region of approximately $2 \mathrm{~mm}$, whereas aggregation fields are usually $1 \mathrm{~cm}$ in diameter. The spiral tip therefore stays within its own aggregation field. After approximately ten rotations, at time $=210 \mathrm{~min}$, the spiral anchors. Stream formation and aggregation occur throughout the process. Spots of high cell density can be seen at the former positions of the spiral wave centre. The spatial pattern depends strongly on the initial amoebae distribution and shows a great amount of variability. For instance, the position at which the spiral anchors varies. Sometimes, the aggregation centre has an empty (no cells present) core around which the spiral rotates. The empty core either disappears after a while, or remains. In addition, the spiral can break up to form several spirals, one of which remains and anchors. Even a double-armed spiral can occur. The occurrence of empty cores, broken or distorted spirals and multiple-armed spirals has also been observed in Dictyostelium aggregation by, for instance, Durston (1973, 1974). The complexity of the spiral wave behaviour is due to the continuously changing excitable medium, caused by chemotaxis.

Another interesting result is that during aggregation, the period of the spiral wave decreases [see Fig. 3(b)]. This feature is also observed in experiments (Gross et al., 1976, 1977; Siegert \& Weijer, 1989). In the model, the period decreases because the wave speed increases with increasing cell density. Therefore, a spiral wave will rotate faster if density increases, resulting in a shorter rotation period. Thus, during aggregation, the increasing cell density in the 
centre results in a decreasing spiral wave period. At the end of the aggregation, the spiral wave period is the same as that at density $100 \%$ [compare Fig. 3(b) and (c)]. Of course, it is still possible that in situ, other processes play a role as well. For instance, the developmentally regulated expression of in vivo adenylate cyclase and phosphodiesterase, which results in faster cAMP and phosphodiesterase production and a shorter refractory period, could also play a role in this process (Gerisch, 1987; Kessin, 1988; Pitt et al., 1992).

A second way to reduce spiral tip wandering in the model is to introduce random motion of the cells by means of the following equation for chemotaxis:

$$
\frac{\mathrm{d} \mathbf{R}_{\mathbf{i}}(t)}{\mathrm{d} t}=\mu \frac{\nabla \gamma(\mathbf{r}, t)}{\|\nabla \gamma(\mathbf{r}, t)\|}+v \mathbf{F}
$$

where $\mathbf{F}$ is a two-dimensional vector containing random numbers between $(-1,1)$ and $v$ is a stepwise function, which equals 0 if condition 1 or 3 is satisfied (see Section 2.1). Otherwise, $v=0.5$. When the model defined by eqns (2) and (5) is used with the parameter values as in Section 2.1, the results are qualitatively the same as in the simulations without random motion and $\varepsilon_{r}=0.71$, i.e. there is stream formation, a decrease in the spiral wave period and a high variety in the spatial patterns.

\section{Experimental Determination of the Wave Speed}

In the model, the dependence of wave speed $(v)$ on cell density $(d)$ is important in stream formation (see Discussion). To gain insight into the $v(d)$ relation in Dictyostelium during aggregation, we performed the following experiment.

(a)

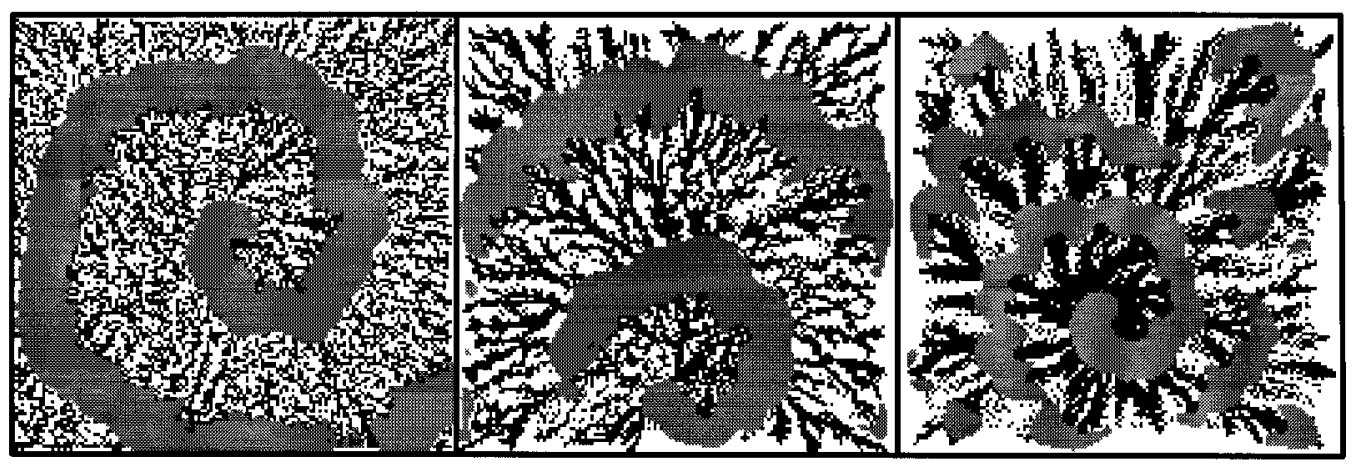

(b)

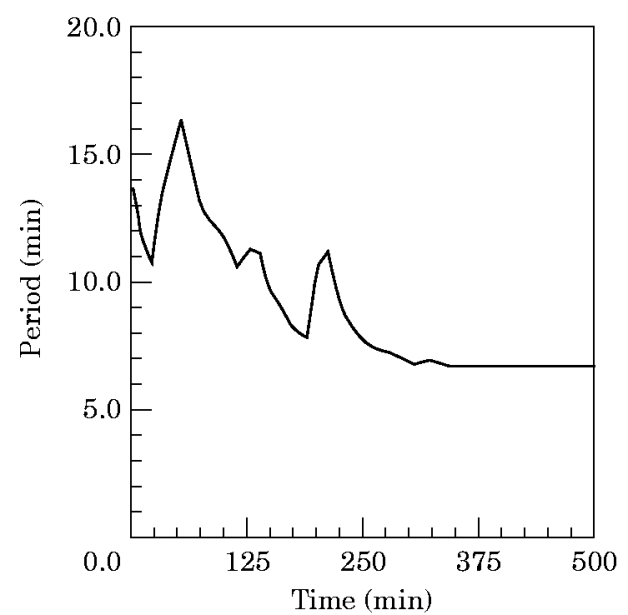

(c)

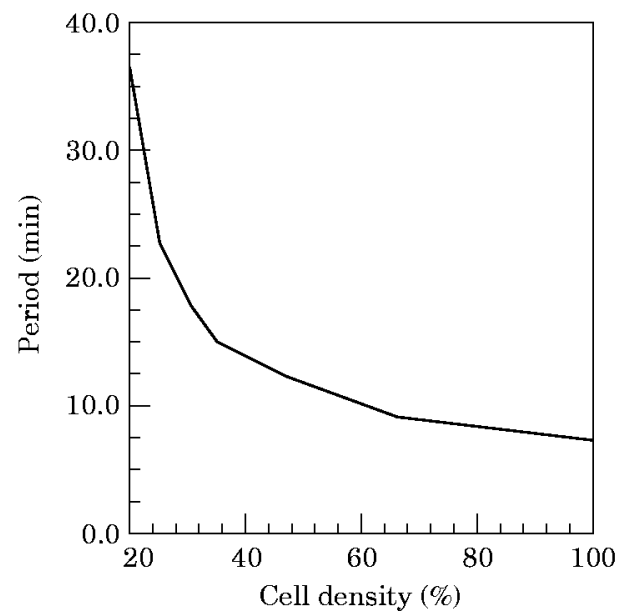

FIG. 3. (a) Aggregation due to a spiral wave in the model defined by eqns (2) and (4). Time $=10,150$ and 450 min, respectively. The spiral is artificially induced by breaking a plane wave; there is no random motion. $\varepsilon_{r}=0.71 ; \mu=20$; field size is $150 \times 150$ mesh points, all other parameters as in Section 2.1. (b) Spiral wave period vs. time in the simulation of Fig. 3(a). The spiral wave period is determined by measuring the time between two cAMP maxima at several assay points in the field. During drift of the spiral the rotation period is dependent on the position of the assay points. However, in all assay points, a global decrease in the spiral period is found. A typical assay point is shown here (peaks are caused by the drift of the spiral). (c) Spiral wave period vs. cell density in the parameter setting of Fig. 3(a). For several values of the cell density $(100,65,45,35,30,25$ and $20 \%)$, the following numerical experiment is done. Cells are randomly distributed with a given density, chemotaxis is blocked $(\mu=0)$ and a spiral is artificially induced by breaking a plane wave. The period of the spiral wave is determined by measuring the time between two cAMP maxima at an assay point in the field. Since the spiral is very stable due to the absence of chemotaxis, one assay point for measuring the spiral wave period is sufficient. 
TABLE 1

Average wave speed and standard deviation at high and low cell density

\begin{tabular}{lccc}
\hline $\begin{array}{l}\text { Wave speed } \\
90-100 \% \text { cells } \\
(\mu \mathrm{m} / \mathrm{min})\end{array}$ & $\begin{array}{c}\text { Wave speed } \\
30-40 \% \text { cells } \\
(\mu \mathrm{m} / \mathrm{min})\end{array}$ & Statistics & $\begin{array}{c}\text { Relative } \\
\text { decrease in } \\
\text { wave speed }(\%)\end{array}$ \\
\hline $303 \pm 121$ & $247 \pm 100$ & $\mathrm{t}=6.63 ; \mathrm{df}=28 ;$ & $18 \pm 12$ \\
& $\mathrm{p}<0.001$ & \\
\hline
\end{tabular}

This table shows the data from seven different experiments (5-10 waves in each experiment). The first column shows average wave speed plus standard deviation at high density (90-100\% area occupancy), the second column average wave speed and standard deviation at low density (30-40\% area occupancy). The difference in wave propagation speeds is highly significant according to the paired students t-test (third column, $\mathrm{df}=$ degrees of freedom, $\mathrm{p}=$ probability of the distributions being the same). The fourth column shows the mean relative decrease in wave speed when the waves go from high to low density, calculated as (first column - second column)/first column, plus standard deviation.

\subsection{METHOD}

Axenic cells $(A \times 2)$ were cultivated according to standard procedures. For all experiments they were grown to densities between $3 \times 10^{6}$ and $6 \times 10^{6}$ cells $\mathrm{ml}^{-1}$. The cells were harvested by low speed centrifugation $(500 \times g)$, washed twice in $20 \mathrm{mM}$ Potassium phosphate buffer, $\mathrm{pH} 6.8$ (KK2) and resuspended at $10^{7}$ cells $\mathrm{ml}^{-1}$ on KK2 buffer. Aggregation plates were prepared by pouring $10 \mathrm{ml} \mathrm{KK} 2$ agar (1\% agar in KK2) onto plates ( $9 \mathrm{~cm}$ diameter) and the cells were plated at densities of $1-3 \times 10^{7}$ cells per plate. The cells were allowed to settle and the excess fluid was decanted. In the middle of the plate one or two drops of high cell density $\left(1 \mu 1\right.$ of $10^{8}$ cells $\left.\mathrm{ml}^{-1}\right)$ were applied and incubated for $4-5 \mathrm{hr}$ at $22^{\circ} \mathrm{C}$. Thereupon the plates were quickly air dried by being placed for 3-5 min in a sterile hood with the blower set at full power. To improve the stability and visibility of the waves $2 \mathrm{mM}$ caffeine was added to the agar. Optical density measurements were performed with a macroscopic darkfield as described in Siegert \& Weijer (1989). The darkfield images were observed with a CCD video camera and digitized in $512 \times 512$ pixels with grey values in the range of 0-255 with the aid of an Imaging Technology image processing board (AFG) and were then analysed with special image analysis programs (Siegert \& Weijer, 1989).

\subsection{RESULTS}

Darkfield waves always appeared first in the regions of high density [Fig. 4(a)] in the form of spirals as is typical for strain $A \times 2$. The waves then propagated from regions of high density into regions of low density, where the wave speed decreased. Depending on the cell density the waves either continued to propagate or eventually disappeared. To analyse the changes in wave speed quantitatively we used a time-space plot, which allows several parameters of the wave propagation process to be analysed simultaneously [Fig. 4(b)]. The time-space plot was constructed by reducing the grey values in a rectangular area of pixels [Fig. 4(a)] to a line of grey values which was stored on hard disk. The grey values were averaged over the height of the rectangle in order to reduce the noise of the video signal. This process was repeated every $10 \mathrm{~s}$ for $500-1000$ successive time points. The time-space plot is shown in Fig. 4(b), where time goes from top to bottom. Propagating waves appear as alternating dark and light bands. The waves originate in the region of high cell density and point downwards into the regions of low cell density. The tangent to these lines indicates the speed of the wave. The analysis of several experiments showed that the waves consistently slow down as soon as they arrive in the region of low cell density. This is visualized in Fig. 4(b) where the tangent of the waves is shown in both regions. The difffrence in the two slopes clearly indicates the

TABLE 2

Wave speed of successive waves in one experiment at high and low cell density

\begin{tabular}{lcc}
\hline $\begin{array}{l}\text { Wave speed } \\
90-100 \% \text { cells } \\
(\mu \mathrm{m} / \mathrm{min})\end{array}$ & $\begin{array}{c}\text { Wave speed } \\
30-40 \% \text { cells } \\
(\mu \mathrm{m} / \mathrm{min})\end{array}$ & $\begin{array}{c}\text { Relative } \\
\text { decrease in } \\
\text { wave speed }(\%)\end{array}$ \\
\hline 415 & - & - \\
460 & 387 & 16 \\
527 & 411 & 22 \\
581 & 339 & 42 \\
581 & 315 & 46 \\
456 & 290 & 37 \\
\hline
\end{tabular}

Wave speed of the successive waves seen in Fig. 4(b) at high cell density (90-100\% area occupancy, first column) and low cell density ( $30-40 \%$ area occupancy, second column), as well as the relative decrease in speed when the waves go from high to low density, calculated as (first column - second column)/first column (third column). 


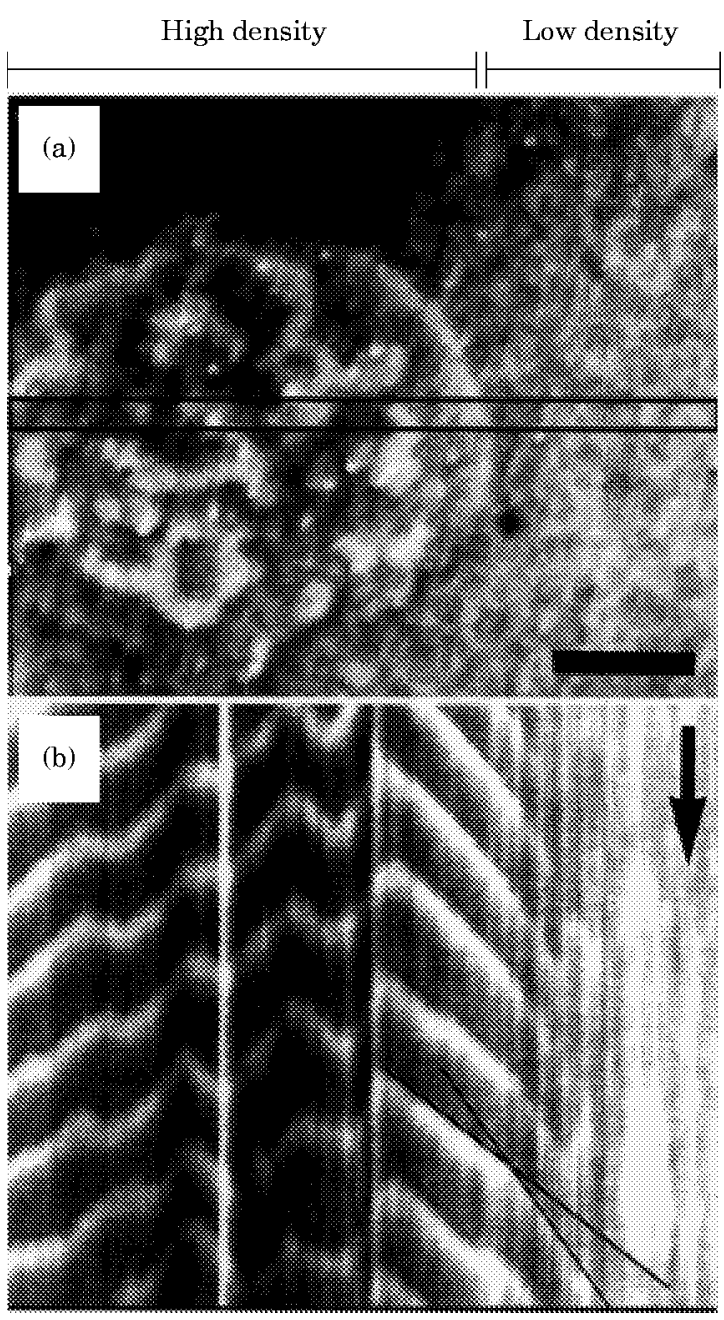

FIG. 4. Wave propagation in fields of high and low cell density in the experiment of Section 3. (a) Darkfield image of an aggregation field. (b) The corresponding time-space plot. The rectangle in Fig. 4(a) indicates the position of the line which was stored at successive time points in order to construct the time-space plot in Fig. 4(b). The black lines in Fig. 4(b) indicate the tangents which were set to determine the wave speed. Bar $3 \mathrm{~mm}$.

change in wave speed. In general there is a reduction of $15-20 \%$ in wave speed as the wave travels from high density (90-100\% area occupancy) to low density (30-40\% area occupied). Table 1 summarizes the speeds of all the waves in seven independent experiments. Table 2 shows the decrease in the wave speed of successive waves in the experiment shown in Fig. 4(b). Statistical analysis of the data in Table 1 shows that the decrease in wave speed is statistically highly significant, despite the large standard deviation of the data. This high variation is due to the continuous drop in wave speed as the cells develop during the aggregation phase, as we have shown previously (Siegert \& Weijer, 1989). The difference in developmental age can be seen in Fig. 4(a). The waves start in the region of high density; there are no waves in the region of low density. The first wave is not yet able to propagate into the low density region, since in this region the cells are not yet able to propagate the signal. The next waves can propagate further into the low density region, but finally also die out. However, before the waves die out they slow down significantly. We observed that wave speed always slows down when a wave travels from a region of high cell density to a region of low cell density; we have never observed an increase in wave speed.

\section{Discussion}

Previously, most differential equation models of the aggregation phase of Dictyostelium discoideum have described the cAMP dynamics either in two (Vasiev et al., 1994; Höfer et al., 1995a, b) or in many (Monk \& Othmer, 1990; Tang \& Othmer, 1994) equations. The first type of model captures the phenomenon of the travelling cAMP waves in a very simple way, whereas the latter type of model aims to describe the biochemical pathway that leads to cAMP production in more detail.

On the other hand, Martiel \& Goldbeter (1987) and Tyson et al. (1989) studied aggregation both by a relatively simple two-equation model and a more elaborate three-equation model, the difference between the two being the inclusion of intracellular cAMP in the model. In the two-equation model, it is assumed that the internal cAMP dynamics is so fast that it can be thought of as at quasi-steady-state, and therefore it is not explicitly included in the equations. Martiel \& Goldbeter (1987) and Tyson et al. (1989) found that the results of the three- and two-equation models differ only qualitatively. By including chemotaxis, we have extended the Martiel-Goldbeter model. It turns out that in this extended model [wherein the same experimentally determined parameters as in Martiel \& Goldbeter (1987) and Tyson et al. (1989) are used], the results of the two- and the three-equation models do differ qualitatively, namely with respect to the spatial cell distribution. In the three-equation model, the cells aggregate but do not form streams, whereas they do form streams in the two-equation model (see Fig. 1). However, when in the three-equation model the turnover rate of intracellular cAMP is increased, streams do form [this result is not dependent on whether the cells move according to eqn (3) or to eqn (4)]. Thus, in this model, the turnover rate of intracellular cAMP is important in stream formation.

In the models on Dictyostelium aggregation by Kessler \& Levine (1993), Vasiev et al. (1994), and Höfer et al. (1995a, b), the internal cAMP dynamics 
is assumed to be instantaneous. Cell streaming was found to occur in all three models. In the light of our model results, the question arises whether the assumption on the very fast internal cAMP dynamics is crucial to the cell streaming found in these models. Both Kessler \& Levine (1993) and Höfer et al. (1995a, b) performed linear stability analysis on an analytic caricature of their models, and came to the conclusion that cell streaming is due to a chemotaxisdriven wavefront instability. Then the question becomes more specified: what role would the turnover rate of $\mathrm{CAMP}_{\text {[in] }}$ play in the stability criterion, if such a stability analysis were made of a model in which internal cAMP dynamics is not assumed to be instantaneous? Interesting in this regard is that Cohen \& Robertson (1971) showed analytically that in their model of $D d$ aggregation, the limiting factor in the wave speed is the intracellular delay, and not intercellular diffusion.

Another way to explain the observed difference in the results of the two- and the three-equation models is the following. One of the differences between the case where $\mathrm{cAMP}_{[\mathrm{in}]}$ dynamics is fast (the streaming case) and the case where it is slow (no stream formation) is the wave speed/cell density relation, $v(d)$. If the wave speed $(v)$ depends on the density of amoebae $(d)$ [as in Fig. 1(c); dotted line], a streaming pattern arises [Figs 1(b) and 2(b)]. On the other hand, if the wave speed does not depend on the cell density [Fig. 1(c); solid line], streams do not form [Figs 1(a) and 2(a)]. Our hypothesis is that streams form due to the density-dependentness of the wave speed. This view is supported by the following arguments. (i) In our simulations, streams occur only if wave speed depends on the density of amoebae. (ii) Vasiev et al. (1994) demonstrated that, in their model, streaming is caused by the density-dependentness of the wave speed. Although their model is more simplified than ours (Fitzhugh-Nagumo equations describe the cAMP waves, cell density is continuous), their wave speed-cell density curve is quite similar to ours. In their case, the relative difference of the wave speed at $100 \%$ and $40 \%$ density $[(v(100 \%)-v(40 \%)) /$ $v(100 \%)]$ is approximately $40 \%$, whereas in our case this difference is approximately $30 \%$. Therefore, we think it is reasonable to assume that in our model, streams also occur due to the density-dependent wave speed.

An informal way to understand how a wave of which the speed is density-dependent could cause stream formation is the following. If the wave travels faster in regions with high cell density, a local difference in the cell density will lead to a local curvature of the wavefront. Imagine for instance a plane wave, passing through a low density region, with a spot of high density in the middle (such a numerical experiment is indeed done by Vasiev et al., 1994). The wave travels faster where density is higher, causing an outward bulge of the wavefront. Cells move orthogonal to the wavefront, and therefore the bulge causes them to move to the regions with higher cell density, thereby further increasing the local density. This will enhance the local curvature of the next wave, thereby attracting more amoebae, and so forth. Thus, streams can form due to minor local differences in the initial density, these differences being enhanced by each successive wave. In the later stages of aggregation, the local curvature of a wave travelling through a stream will be so strong [see Fig. 1(b) at $t=170 \mathrm{~min}$ ] that cells in the neighbourhood of the stream will move more in the direction of the stream than towards the aggregation centre, a phenomenon which is also observed in situ (Siegert \& Weijer, unpublished observations). In the case where $\mathrm{cAMP}_{[\mathrm{in}]}$ dynamics is slow and wave speed hardly depends on density, a local difference in the cell density does not cause a local curvature of the wavefront. Then all concentric waves remain completely circular and amoebae move directly to the aggregation centre, without forming streams.

If the aggregation field is thought of as a standard excitable medium, it is reasonable to assume that high cell density corresponds to high excitability. In this case, it is to be expected that wave speed increases with density, since waves travel faster in regions of high excitability. High cell density corresponding to high excitability was implemented by Kessler \& Levine (1993), Vasiev et al. (1994) and Höfer et al. (1995a, b). Indeed, both Kessler \& Levine (1993) and Vasiev et al. (1994) reported that in their models, wave speed increases with density, similar to our Fig. 1(c) (dotted line). In Höfer et al. (1995a, b) the wave speed/cell density curve was not measured. In contrast to the three previously mentioned models, Monk \& Othmer (1990) and Tang \& Othmer (1994) did not assume fast internal cAMP dynamics. In Monk \& Othmer (1990) a $v(d)$ curve was found which differs from those in Vasiev et al. (1994), Kessler \& Levine (1993) and from ours [the $v(d)$ curve was not measured in Tang \& Othmer (1994)]. Wave speed in the Monk-Othmer model is high at low density and vice versa. Unfortunately, since chemotaxis was included neither in Monk \& Othmer (1990) nor in Tang \& Othmer (1994), it is not known whether streams would form in these models.

Experimental evidence concerning the relation between wave speed and cell density is very scarce. Therefore, we investigated the wave speed at two 
different cell densities to get an idea of the $v(d)$ relation in Dictyostelium discoideum. We found that wave speed always decreases when the wave travels from high (90-100\% occupancy) to low density (30-40\% occupancy), and that below a critical density $(10 \%)$ the waves disappear. This indicates that wave speed depends on cell density. The decrease in wave speed is less than in the model (the relative decrease in the wave speed in Table 1 is $18 \%$, as opposed to $30 \%$ in the model), but in both cases the wave speed decreases. A complication of the experiment is that cells at high density develop faster than cells at low density. Since wave speed has been shown to decrease during aggregation, and thus during development (Siegert \& Weijer, 1989), this might well influence the results. This decrease in wave speed during aggregation could be due to developmental changes within the cells that affect their relaying properties. Another possibility is that the wave period decrease during aggregation causes a decrease in wave speed by pushing the system down the dispersion curve [see for instance Höfer et al. $(1995 \mathrm{a}, \mathrm{b})]$. If wave speed decrease during aggregation is solely due to the period decrease (which, in turn, could be caused entirely by the increase in cell density in the aggregation centre, see Section 2.2), the previously mentioned complication is of minor importance. However, if wave speed would depend on the developmental stage of the cells, this would cause the wave in the region of high cell density to travel more slowly than expected, thereby reducing the difference in wave speed at the different cell densities. This indicates that under normal aggregation conditions, the difference in cell speed between high and low density regions might be larger than found in our experiment.

In contrast to our experimental results, Alcantara \& Monk (1974) reported that wave speed slightly decreases as cell density increases. However, in their experiments, the developmental stage of the cells in the different densities was not considered. Moreover, in their experiment in which the difference in wave speed at high and low density is statistically significant, the period of cAMP stimulation is not taken under consideration. Still, in the same paper it is shown that the period of stimulation in their experiments varies substantially, which might very well influence the results (in our experimental set up, cells in high and in low density are subject to the same stimulation period). To solve the contradiction in experimental results concerning the relation between cell density and wave speed, new and more detailed experimental evidence is needed. In such experiments, special care will have to be given to compare cells under the same period of stimulation and in the same developmental stage.

On the basis of our model, and keeping in mind its perhaps unrealistic assumption about the fast internal cAMP dynamics, we expect a stronger densitydependence of the wave speed if the streams formed by this specific strain are very pronounced, whereas we expect a much weaker dependence in the case of weak stream formation. In addition, the model predicts that Dictyostelium mutants that have very slow intracellular cAMP dynamics will not be able to form streams. New experimental evidence is needed to test these hypotheses.

We thank Mrs. McNab for linguistic advice.

\section{REFERENCES}

Alcantara, F. \& Monk, M. (1974). Signal propagation during aggregation in the slime mould Dictyostelium discoideum. J. Gen. Microbiol. 85, 321-334.

COHEN, M. \& RoberTson, A. (1971). Wave propagation in the early stages of aggregation of cellular slime molds. J. theor. Biol. 31, 101-108.

Devreotes, P. (1982). Chemotaxis. In: The Development of Dictyostelium discoideum. (Loomis, W. ed.), pp. 117-168. New York: Academic Press.

Devreotes, P. (1989). Dictyostelium discoideum: a model system for cell-cell interactions in development. Science 245, 1054-1058.

Devreotes, P. \& Zigmond, S. (1988). Chemotaxis in eukaryotic cells: a focus on leukocytes and Dictyostelium. Ann. Rev. Cell Biol. 4, 649-686.

Durston, A. (1973). Dictyostelium discoideum aggregation fields as excitable media. J. theor. Biol. 42, 483-504.

Durston, A. (1974). Pacemaker activity during aggregation in Dictyostelium discoideum. Develop. Biol. 37, 225-235.

Fisher, P., MERKL, R. \& Gerisch, G. (1989). Quantitative analysis of cell motility and chemotaxis in Dictyostelium discoideum by using an image processing system and a novel chemotaxis chamber providing stationary chemical gradients. J. Cell Biol. 108, 973-984.

GERISCH, G. (1987). Cyclic amp and other signals controlling cell development and differentiation in Dictyostelium discoideum. Annu. Rev. Biochem. 56, 853-879.

Gross, J., Kay, R., Lax, A., Peacey, M., Town, C. \& Trevan, D. (1977). Cell contact, signalling and gene expression in Dictyostelium. In Development and differentiation in the cellular slime moulds. (Cappuccinelli, P. and Ashworth, J. eds), pp. 135-147.

Gross, J., Peacey, M. \& Trevan, D. (1976). Signal emission and signal propagation during early aggregation in Dictyostelium discoideum. The Cell Sci. 22, 645-656.

Höfer, T., Maini, P. K., Sherratt, J. A., Chaplain, M., Chauvet, P., Metevier, D., ET AL. (1994). A resolution of the chemotactic wave paradox. Appl. Math. Lett. 7(2), 1-5.

Höfer, T., SherratT, J. A. \& Maini, P. K. (1995a). Cellular pattern formation during Dictyostelium aggregation. Physica D 85, 425-444.

HöFer, T., Sherratt, J. A. \& Maini, P. K. (1995b). Dictyostelium discoideum: cellular self-organization in an excitable biological medium. Proc. Roy. Soc. Lond. B 259, 249-257.

KeLLER, E. \& SEgEL, L. (1970). Initiation of slime mold aggregation viewed as an instability. J. theor. Biol. 26, 399-415.

Kessin, R. (1988). Genetics of early Dictyostelium discoideum development. Microbiol. Rev. 52, 29-49. 
Kessler, D. \& Levine, H. (1993). Pattern formation in Dictyostelium via the dynamics of cooperative biological entities. Phys. Rev. E 48, 4801-4804.

LeVINE, H. \& ReYNolds, W. (1991). Streaming instability of aggregating slime mold amoebae. Physical Review Letters 66, 2400-2403.

Loomis, W. (1982). The Development of Dictyostelium discoideum. New York: Academic Press.

MACKAY, S. (1978). Computer simulation of aggregation in Dictyostelium discoideum. J. Cell Sci. 33, 1-6.

Martiel, J. \& Goldbeter, A. (1987). A model based on receptor desensitization for cyclic AMP signalling in Dictyostelium cells. Biophys. J. 52, 807-828.

Monk, P. \& OTHMER, H. (1990). Wave propagation in aggregation fields of the cellular slime mould Dictyostelium discoideum. Proc. Roy. Soc. Lond. B 240, 555-589.

Newell, P. \& Liu, G. (1992). Streamer F mutants and chemotaxis of Dictyostelium. BioEssays 14, 473-479.

Parnas, H. \& Segel, L. (1977). Computer evidence concerning the chemotactic signal in Dictyostelium discoideum. J. Cell Sci. 25, 191-204.

Parnas, H. \& Segel, L. (1978). A computer simulation of pulsatile aggregation in Dictyostelium discoideum. J. theor. Biol. 71, 185-207.

Pitt, G., Milona, N., Borleis, J., Lin, K., Reed, R. R. \& Devreotes, P. N. (1992). Structurally distinct and stage-specific adenylyl cyclase genes play different roles in Dictyostelium development. Cell 69, 305-315.

Siegert, F. \& WeiJer, C. (1989). Digital image processing of optical wave propagation in Dictyostelium discoideum and analysis of the effects of caffeine and ammonia. J. Cell Sci. 93, $325-335$.
Steinbock, O., Hashimoto, H. \& MüLler, S. (1991). Quantitative analysis of periodic chemotaxis in aggregation patterns of Dictyostelium discoideum. Physica D 49, 233-239.

TANG, Y. \& Othmer, H. (1994). A G Protein-based model of adaptation in Dictyostelium discoideum. Math. Biosci. 120, 25-76.

Tomchiк, K. \& Devreotes, P. (1981). Adenosine 3', 5'monophosphate wave in Dictyostelium discoideum: a demonstration by isotope dilution-fluorography. Science $\mathbf{2 1 2}$ 443-445.

Tyson, J., Alexander, K., Manoranjan, V. \& Murray, J. (1989). Spiral waves of cyclic AMP in a model of slime mold aggregation. Physica D 34, 193-207.

Van De Merwe, A. P. (1995). Indications for simple mechanisms underlying aggregation patterns in Dictyostelium discoideum. Master's thesis, Theoretical Biology, Utrecht University, Padualaan 8, NL-3584 CH, Utrecht, the Netherlands (Series in Bioinformatics 95:4:10).

Varnum-Finney, B., Schroeder, N. \& Soll, D. (1988). Adaptation in motility response to camp in Dictyostelium discoideum. Cell Motil. Cytoskel. 9, 9-16.

Vasiev, B., Hogeweg, P. \& Panfilov, A. (1994). Simulation of Dictyostelium discoideum aggregation via reaction-diffusion model. Physical Review Letters 73, 3173-3176.

Vasieva, O., Vasiev, B., Karpov, V. \& Zaikin, A. (1994). A model of Dictyostelium discoideum aggregation. J. theor. Biol. 171, 361-367.

Wessels, D., Murray, J. \& Soll, R. (1992). Behaviour of Dictyostelium amoebae is regulated primarily by the temporal dynamic of the natural cAMP wave. Cell Motil. Cytoskel. 23, 145-156.

Wilkinson, P. \& Haston, W. (1988). Chemotaxis: an overview. Methods in Enzymology 162, 3-16. 